\title{
Synthesis of solvent-modified epoxies via Chemically Induced Phase Separation: A new approach towards void toughening of epoxies
}

\author{
J. Kiefer, H. H. Kausch, J. G. Hilborn* \\ Swiss Federal Institute of Technology, Materials Department, Polymers Laboratory, \\ CH-1015 Lausanne, Switzerland
}

Received: 23 October 1996/Revised version: 6 January 1997/Accepted: 23 January 1997

\section{Summary:}

The fracture toughness has been investigated with single edge notched bending specimens in solvent-modified epoxies, which were prepared via the Chemically Induced Phase Separation technique. The generation of a controlled morphology with liquid droplets in the micrometer range leads to a substantial increase in the fracture energy of nearly $400 \%$ compared to the non-modified epoxy network. The critical stress intensity factor of these highly crosslinked thermosets does not vary significantly. These results demonstrate the general potential of the Chemically Induced Phase Separation technique to prepare porous thermosets, thus combining increased toughness with lowered density.

\section{Introduction:}

Epoxies are among the most widely used thermosetting polymers. The wide variety of curing agents and epoxies with different chemical structures allows for the synthesis of tailored polymers which can fulfill special demands for advanced applications. These polymers have therefore found widespread use in electronic and optical packaging materials, as adhesives, coatings, and as matrix materials for high performance composites (1). Despite their favorable properties, epoxies are inherently brittle as a consequence of the formation of a highly crosslinked network structure. The brittleness can be overcome by the incorporation of second phase particles (2-6). Even though this strategy is a well established method since more than two decades, there exists still a controversal discussion concerning the morphological features for most effective toughening. The nature of the dispersed phase does not seem to play the predominant role for toughening. A wide variety of thermoplastic $(7,8)$ or rubbery particles $(9)$, that are generated via reaction induced phase separation have been used for theses purposes. The thermodynamic origins of the phase separation process and the influence of internal and external reaction parameters has been studied extensively for numerous types of thermoplastic $(10,11)$ and rubber (12-16) modified epoxies as well as for other classes of thermosets such as cyanurates (17). This allows to control the morphology and to optimize the mechanical properties (18). However the generation of a two phase structure by phase separation leads often to a reduction in thermal stability. A considerable improvement in toughness can be achieved by using highly branched polymers, phase separating to dispersed domains (19). Alternatively, the desired toughening effect can be obtained by blending with core shell particles (20-25). The degree of agglomeration of this particles can be adjusted by a surface modification. Conversely to previous strategies to achieve a maximum dispersion of core-shell particles, very recent investigations show better toughenability, if no surface modification is realized (20). The higher toughness is attributed to a larger plastic zone size, which is observed in systems clearly showing interconnected particles. In contrast to its nature, the size and distribution of the second phase particles seems to be crucial for effective 
toughening. Several research groups observed, that no toughening occurs, if the second phase particles are smaller than $150-200 \mathrm{~nm}(24,26)$. Theoretical considerations predict, that this is a lower critical size to induce cavitation (27). It could be proven with scattering techniques, that the cavitation preceeds the formation of shear bands $(26,28$, 29 ), which is recognized as the most important toughening mechanism $(9,30)$. Furthermore, the contribution of the plastic void growth, succeeding cavitation, to the total toughness can become as important as shear banding, especially at elevated temperatures (31). Cavitation is equal to the generation of a series of voids. Indeed, such a morphology has been simulated by using either a non reactive rubber (32) or hollow latex spheres $(33,34)$ as the dispersed phase. In the first study no insight was given in the removal of the liquid rubber and only one single composition has been studied. In the second study, hollow particles were used, thus creating an additional interface. Using such pseudo-porous systems, both groups indicated the ability of voids to toughen epoxies in the same manner and in the same magnitude as rubber particles. The same group calculated the stress distributions of spherical inclusions, being either rubber or voids, in an isotropic epoxy matrix based on a finite element model $(31,35-37)$. It was concluded, that the effect of voids or a rubbery phase is very similar. These inclusions can release the degree of triaxial stresses at the crack tip. Even though, this model allowed to predict the possibility of void toughening, the finite element approach does not allow to take into account the multiple interactions between voids. Therefore a refined model has been developed, which is based on a zero order approximation and allows to visualize the stress distributions in porous epoxies $(38,39)$. This model predicts the buildup of internal stresses which can initiate multiple shear banding, thus leading to effective toughening. A better understanding of the cavitation process is crucial to develop new materials, combining high specific properties with increased toughness. Despite all the above reports, the cavitation is still described as a side phenomen occuring during the deformation of toughened epoxies (40).

The experimental verification of the above theoretical predictions of void toughening requires a technique which yields porous thermosets having closed pores with sizes and distributions in the $\mu \mathrm{m}$-range similar to those commonly used for toughening with rubber or thermoplastic particles. Therefore, we have developed a new technology, termed Chemically Induced Phase Separation (CIPS) $(39,41-44)$, for the generation of the desired morphology which is governed by a phase separation process resulting from a chemical quench (45). With this method, the epoxy precursor and curing agent are cured in the presence of a low molecular weight liquid, which turns into a non-solvent upon curing, thus initiating a phase separation proceeding via a nucleation and growth mechanism. This process results in the formation of liquid droplets, spherical in shape due to thermodynamic reasons. The generation of a porous morphology is subsequently achieved by diffusion of the liquid through the crosslinked matrix without any significant alteration in the size and distribution of the dispersed phase. This new type of macroporous thermosets is characterised by a very narrow size distribution in the $\mu \mathrm{m}$ range and a significant lower density without any lowering in thermal stability. In a previous study (43) we have investigated the role of the reaction parameters such as the chemical nature of the solvent, its concentration and the curing temperature, thus allowing for morphology control.

It is the purpose of this paper to present the results of our first studies concerning the evaluation of the fracture toughness of solvent modified epoxies which were prepared by the CIPS technique. These investigations are intended to throw some light on the ongoing discussion on the role of the second phase and cavitation for the toughening of 
thermosets. Indeed, we believe, that rubbery particles, liquids or even voids can cause the desired stress distribution, thus leading to effective toughening.

\section{Experimental:}

Materials:

Bisphenol A diglycidylether (DER332 from Fluka) and 2,2-bis(4-amino-cyclohexyl) propane (HY2954 from Ciba-Geigy) were used as the precursors to build the epoxy network. Cyclohexane was purchased in analysis grade.

\section{Sample preparation:}

Macroporous epoxies were prepared according to the CIPS technique as described below. First the bifunctional epoxy precursor was mixed at room temperature with the tetrafunctional diamine in a stochiometric ratio (2:1). This mixture was then degassed in a vacuum oven at room temperature. After degassing, cyclohexane was added under slight stirring in the desired amount to yield a homogeneous, transparent mixture. This homogeneous mixture was then transferred into steel molds designed to yield plates with a thickness of $4 \mathrm{~mm}$. The molds were placed into an oven at $40^{\circ} \mathrm{C}$ for $16 \mathrm{~h}$, thus allowing for curing and in situ phase separation. Then the oven temperature was raised stepwise with $20 \mathrm{~K} / \mathrm{h}$ to $200^{\circ} \mathrm{C}$ to allow for partial solvent removal and full cure. Then the samples were allowed to cool to room temperature in the closed oven for further $16 \mathrm{~h}$ to avoid macroscopic crack formation. After removing from the mold, bars with $w=8 \mathrm{~mm}, b=4$ $\mathrm{mm}$ and $\mathrm{L}=73 \mathrm{~mm}$ in size were machined from the resulting plates for further characterizations.

\section{Characterization methods:}

The critical stress intensity factor, $\mathrm{K}_{\mathrm{Ic}}$, and the fracture energy, $\mathrm{G}_{\mathrm{Ic}}$, were determined on single edge notched bending (SENB) specimens (fig.1) providing plane strain conditions and strictly following the instructions in (46). The precrack is formed by tapping on a razor blade, which was placed into the machined notch and has been immersed previously in liquid nitrogen for around $30 \mathrm{~s}$. A testing speed of $1 \mathrm{~mm} / \mathrm{min}$ has been choosen for all experiments. The crack length $a$ is measured prior to fracture under slight bending with a magnifying glass with integrated scale, thus allowing for an accuracy of $0.1 \mathrm{~mm}$. The distance 1 has been choosen as $32 \mathrm{~mm}$.

Density measurements were performed according to the Archimedes principle.

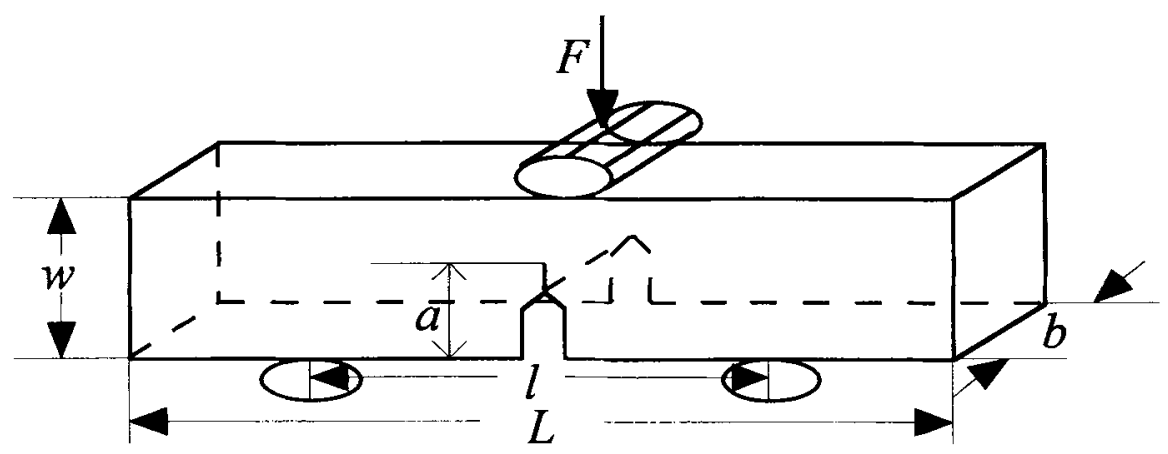

Fig. 1: Single edge notched bending (SENB) specimens used for fracture toughness testing 


\section{Results and discussion:}

When cured at $40^{\circ} \mathrm{C}$ the desired phase separation is achieved, if the initial amount of cyclohexane becomes equal or greater than $14 \mathrm{wt} \%$. The onset of phase separation can be easily detected, as the samples become opaque, thus indicating the formation of a second phase with sizes larger than the wavelength of visible light. Samples with $13 \mathrm{wt} \%$ cyclohexane or less stay transparent. These observations are in perfect agreement with previous results reported in (43). However in those systems, sealed glass tubes were used as reaction vessels, whereas in the present investigation the curing is realized in open molds. Nevertheless a solvent loss of more than $1 \mathrm{wt} \%$ can be excluded as the critical amount for phase separation is identical for both curing procedures. The sample morphologies were checked with scanning electron microscopy (SEM), and no difference concerning the size and distribution of the spherical domains between the two series could be detected. Typical SEM micrographs for different amounts of cyclohexane are shown in figure 2.

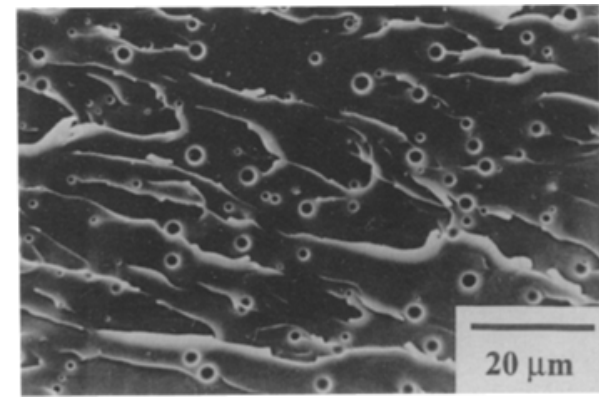

a) 14 wt\% cyclohexane

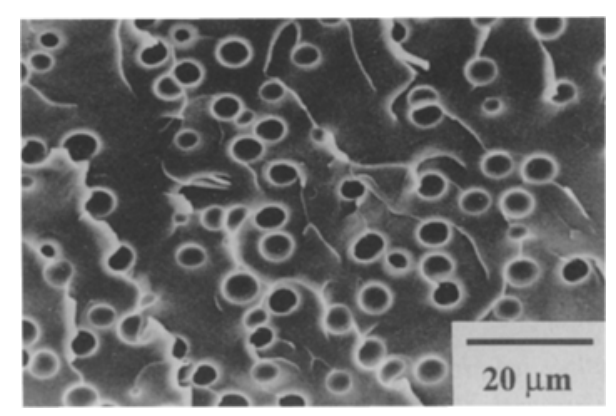

b) $18 \mathrm{wt} \%$ cyclohexane

Fig. 2: SEM micrographs of solvent-modified epoxies prepared via the CIPS-technique with various amounts of cylohexane

Figure 2 shows clearly the increase in domain size and volume fraction associated with the initial amount of solvent. Furthermore the droplets are spherical in shape as a consequence of a nucleation and growth process (43). The morphological characteristics are summarized in table 1 together with the density values after each processing step.

Table 1: Characteristics of epoxies cured at $40^{\circ} \mathrm{C}$ in the presence of various amounts of cyclohexane

\begin{tabular}{|c|c|c|c|c|c|}
\hline $\begin{array}{c}\text { wt\% } \\
\text { cyclohexane }\end{array}$ & $\begin{array}{c}\text { droplets } \\
\text { diameter } \\
(\mu \mathrm{m})\end{array}$ & $\begin{array}{c}\text { volume } \\
\text { fraction } \\
(\%)\end{array}$ & $\begin{array}{c}\text { Density } \\
\text { prior drying } \\
\left(\mathrm{g} / \mathrm{cm}^{3}\right)\end{array}$ & $\begin{array}{c}\text { Density after } \\
\text { post curing } \\
\left(\mathrm{g} / \mathrm{cm}^{3}\right)\end{array}$ & $\begin{array}{c}\text { Density after } \\
\text { complete drying } \\
\left(\mathrm{g} / \mathrm{cm}^{3}\right)\end{array}$ \\
\hline 0 & 0 & 0 & - & - & 1.123 \\
\hline 13 & 0 & 0 & - & 1.089 & 1.121 \\
\hline 14 & 2.3 & 2.8 & 1.083 & 1.083 & 1.092 \\
\hline 15 & 3.4 & 4.5 & 1.076 & 1.079 & 1.072 \\
\hline 16 & 4.1 & 5.2 & 1.073 & 1.075 & 1.065 \\
\hline 18 & 4.6 & 7.7 & 1.064 & 1.07 & 1.037 \\
\hline 20 & 5.4 & 11.8 & 1.033 & 1.066 & 0.991 \\
\hline
\end{tabular}


The density measurements after the post curing clearly indicate that the stepwise heating does not lead to the generation of a truly porous morphology. Thus the two phase system studied here consists of dispersed, liquid droplets in an epoxy network. The cavitation ability depends on the modulus of the inner phase, which is practically neglicable for liquids (47). Therefore these materials, which can be regarded as pseudoporous, are used to study the potential of liquids or alternatively voids for the toughening of epoxies.

In order to ensure a minimum value of the fracture toughness, a sharp crack must be realized. The total crack length, $a$, should be in the range of $0.45<a / w<0.55$. The values of fracture toughness $\mathrm{K}_{\mathrm{Ic}}$ and fracture energy $\mathrm{G}_{\mathrm{Ic}}$ are determined according to equations 1 and 2.

$$
K_{I c}=f \cdot \frac{P}{b \cdot \sqrt{w}} \quad \text { (1) } \quad G_{I c}=\frac{U}{b \cdot w \cdot \Phi}
$$

Therein $P$ represents the maximum force, $b$ and $w$ the sample dimensions as shown in figure 1 and $U$ is the area under the load displacement curve. The machine compliance has been taken into account in order to exactly calculate $U$ and consequently the fracture energy. The calibration factors $f$ and $\Phi$ in equations 1 and 2 are defined in (46) and depend strongly on the ratio of $a / w$. Therefore the exact determination of the crack length $a$ is of uppermost importance to obtain correct values for $\mathrm{K}_{\mathrm{Ic}}$ and $\mathrm{G}_{\mathrm{Ic}}$. A natural crack was initiated by slightly tapping on a razor blade, and the crack length, $a$, was measured on both sides of the sample. For our studies, we took the longest crack length visible at the surface as the value of $a$. This procedure leads automatically to low values for the calibration factor $f$ in equation 1 and high values of $\Phi$ in equation 2 , thus ensuring minimal values of $\mathrm{K}_{\mathrm{Ic}}$ and $\mathrm{G}_{\mathrm{Ic}}$ respectively. The values of the critical stress intensity factor, $\mathrm{K}_{\mathrm{Ic}}$, and the fracture energy, GIc, shown in figure 3 represent mean values on an average of 3 to 6 measurements for each composition and are obtained by strictly following the guidelines of the testing protocol (46). The sample dimensions were checked to fulfill the plane strain conditions in accordance with (46). Furthermore only those samples were taken into consideration, where the value of $\mathrm{K}_{\mathrm{Ic}} \mathrm{c}^{2} / \mathrm{G} \mathrm{Ic}$ does not differ more than $15 \%$ from the corresponding value calculated from the calibration factors and the compliance, thus satisfying the requirements of the testing protocol (46).

The pseudo-porous epoxies prepared with various amounts of cyclohexane show a substantial increase in fracture energy, which increases almost lineary with the amount of cyclohexane above the critical concentration to induce the phase separation (figure 3 ). The solvent remaining in the matrix leads to a plasticization only resulting in a slight increase of the fracture energy as it can be conluded by comparing the non-modified epoxy to the sample prepared with $13 \mathrm{wt} \%$ cyclohexane just below the critical concentration to induce a phase separation. The substantial increase in fracture energy of up to around $400 \%$ compared to the neat matrix is a consequence of the buildup and interaction of internal stresses resulting from the generation of a randomly dispersed phase, as concluded from $(38,39)$, thus enabling extensive shear band formation. Several micromechanisms might be associated with this observation. First of all one could imagine, that the crack is deflected by the liquid droplets. Alternatively, plastic void growth might have occured in the plastic zone at the front of the crack tip, thus contributing to energy absorption. Furthermore the radius of the crack tip becomes larger, as the crack penetrates into the droplets. Ongoing work is dedicated to unravel the detailed micromechanisms responsible for the substantial increase in fracture energy. 


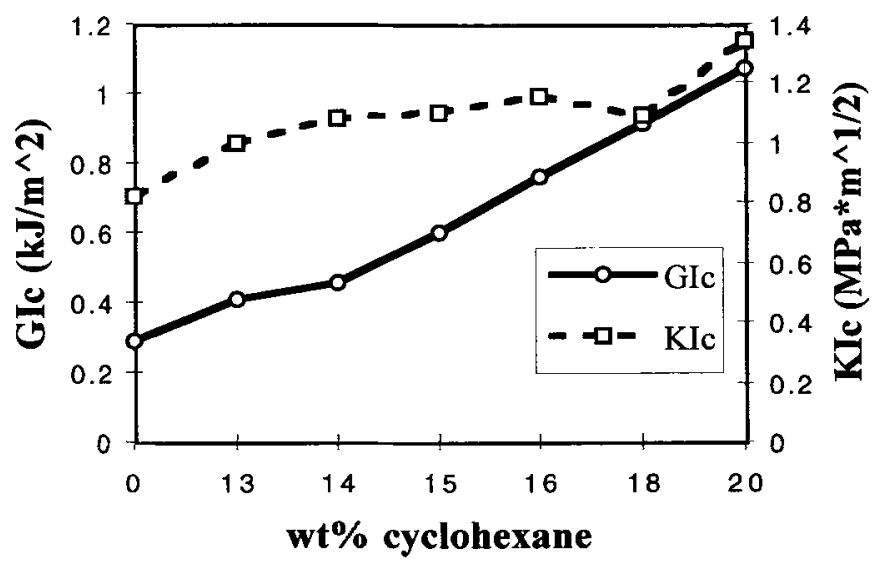

Fig. 3: Fracture energy GIc and critical stress intensity factor KIc of solvent-modified epoxies prepared via the Chemically Induced Phase Separation (CIPS) technique with various amounts of cyclohexane

The epoxy precursor and curing agent choosen for these studies were both low molecular weight components. They were very suitable for our purposes, as they are liquids at room temperature thus allowing for mixing below the boiling point of cyclohexane. On the other hand these precursors lead to a very high crosslink density. It is well known that the toughening effect strongly depends on the molecular weight between the crosslink points $(21-23)$. This is reflected in the results for the critical stress intensity factor (figure 3). A slight improvement in $\mathrm{K}_{\mathrm{Ic}}$ can be concluded from the generation of a dispersed phase, whereas no conclusions concerning the dependency of $\mathrm{KIc}$ on the initial amount of cyclohexane can be drawn from this data, as the experimental error is larger than the variation. To overcome this problem, precusors should be used which yield a lower crosslink density.

\section{Conclusions:}

The synthesis of solvent-modified thermosets via the Chemically Induced Phase Separation technique can lead to a substantial increase in fracture energy even in highly crosslinked epoxies. These results demonstrate, that the toughening depends strongly on the ability of the second phase to generate internal stress concentrations. A further optimization of the drying procedure is required to yield porous structures, thus allowing to evaluate the use of voids for the toughening of thermosets. Therefore the present results provide an intermediate step towards porous thermosets which combine a lowering in density with a significant increase in toughness.

\section{References:}

1. Ellis B (1993) Chemistry and Technology of Epoxy Resins. Black Academic \& Professional, London

2. Arends CB (1996) Polymer Toughening. Marcel Dekker, New York

3. Collyer AA (1994) Rubber toughened engineering plastics. Chapman \& Hall, London

4. Riew CK, Gillham J (1984) Rubber-modified thermoset resins. Adv. Chem. Ser. 208

5. Riew CK (1989) Rubber toughened plastics. Adv. Chem. Ser. 222

6. Riew CK, Kinloch AJ (1993) Toughened Plastics. Adv. Chem. Ser. 233 
7. Hedrick JC, Patel NM , McGrath JE (1993) Adv. Chem. Ser. 233:293

8. Hedrick JL, Yilgor I, Hedrick JC, Wilkes GL, McGrath JE (1991) Polymer 32:2020

9. Sue HJ, Garcia-Meitin EI , Pickelman DM (1996) in Ref. 2:131

10. Oyanguren PA, Williams RJJ, Girard-Reydet E, Pascault JP (1996) Polymer 37:3079

11. Riccardi CC, Borrajo J, Williams RJJ, Girard-Reydet E, Sautereau H, Pascault JP (1996) J. Appl. Polym. Sci. 34:349

12. Williams RJJ, Borrajo J, Adabbo HE, Rojas AJ (1984) Adv. Chem. Ser. 208:195

13. Vazquez A, Rojas AJ, Adabbo HE, Borrajo J , Williams RJJ (1987) Polymer 28:1156

14. Riccardi CC, Borrajo J , Williams RJJ (1994) Polymer 35:5541

15. Verchere D, Sautereau H, Pascault JP, Riccardi CC, Moschiar SM, Williams RJJ (1991) J. Appl. Polym. Sci. 42:701

16. Verchere D, Sautereau H, Pascault JP, Riccardi CC, Moschiar SM, Williams RJJ (1991) J. Appl. Polym. Sci. 42:717

17. Williams RJJ , Pascault JP (1995) Polymer 36:3541

18. Verchere D, Sautereau H, Pascault JP, Riccardi CC, Moschiar SM, Williams RJJ (1991) J. Appl. Polym. Sci. 43:293

19. Boogh L, Pettersson B, Japon S, Månson JAE (1995) Proc. ICCM 10:VI

20. Bagheri R, Pearson RA (1996) J. Mat. Sci. 31:3945

21. Lu AF, Cantwell WJ, Kausch HH (1995) Macromol. Symp. 93:317

22. Lu AF, Plummer CJG, Cantwell WJ , Kausch HH (1996) Polym. Bull. 37:399

23. Lu AF, Kausch HH, Cantwell WJ, Fischer M (1996) J. Mat. Sci. Lett. 15:1018

24. Kim DS, Cho K, Park CE (1996) Polym. Eng. Sci 36:755

25. Sue HJ (1992) J. Mat. Sci. 27:3098

26. Dompas D, Groeninckx G, Hasegawa T, Kadokura M (1994) Polymer 35:4750

27. Dompas D, Groeninckx G (1994) Polymer 35:4743

28. Bubeck RA, Buckley DJ, Jr., Kramer EJ, Brown HR (1991) J. Mat. Sci. 26:6249

29. Lazzeri A, Bucknall CB (1993) J. Mat. Sci. 28:6799

30. Huang Y, Hunston DL, Kinloch AJ, Riew CK (1993) Adv. Chem. Ser. 233:1

31. Huang Y, Kinloch AJ (1992) J. Mat. Sci. Lett. 11:484

32. Huang Y, Kinloch AJ (1992) Polymer 33:1330

33. Bagheri R, Pearson RA (1993) 25th Int. SAMPE Techn. Conf. 25

34. Bagheri R, Pearson RA (1995) Polymer 36:4883

35. Huang Y, Kinloch AJ (1992) J. Mat. Sci. 27:2763

36. Guild FJ, Kinloch AJ (1995) J. Mat. Sci. 30:1689

37. Bucknall CB, Karpodinis A, Zhang XC (1994) J. Mat. Sci. 29:3377

38. Fond C, Kiefer J, Mendels D, Ferrer JB, Kausch HH, Hilborn JG, in preparation

39. Kiefer J, Porouchani R, Mendels D, Ferrer JB, Fond C, Hilborn JG, Kausch HH (1996) Oberflächen Werkstoffe 5:12

40. Cruz CA, Jr., Havriliak S, Jr. (1995) ANTEC 1521

41. Kiefer J, Hilborn JG, Hedrick JL, Cha HJ, Yoon DY, Hedrick JC Macromolecules, to appear

42. Kiefer J, Hilborn JG, Månson JAE, Leterrier Y, Hedrick JL (1996) Macromolecules 29:4158

43. Kiefer J, Hilborn JG, Hedrick JL (1996) Polymer 37 (25):5715

44. Kiefer J, Porouchani R, Mendels D, Ferrer JB, Fond C, Hedrick JL, Kausch HH, Hilborn JG (1996) in MRS Symp. Proc. 431 "Microporous and Macroporous Materials":527

45. Pascault JP (1995) Macromol. Symp. 93:43

46. (1990) Testing protocol LEFM Standard, ESIS, Polymer \& Composites Task group.

47. Liang H, Jiang W, Zhang J, Jiang B (1996) J. Appl. Polym. Sci. 59:505 